\title{
Ser una wianbu en la Corea del Sur de la posguerra
}

\section{Being a Wianbu in Post-war South Korea}

\author{
MORGAN MOK-WON PARK \\ Universidad de Salamanca, España
}

Resumen: El artículo compara dos textos que ofrecen distintas perspectivas sobre una misma situación de opresión ejercida sobre las mujeres desde el poder. En primer lugar, examina un informe emitido por la policía del distrito de Yongsan de Seúl en 1971. Se trata de unas directrices que, con un lenguaje retórico, pretendían condonar los crímenes que los soldados americanos cometían contra las prostitutas al mismo tiempo que trataban de imponer a la población coreana una actitud benévola hacia aquéllos. Paralelamente, el artículo analiza la novela Chinatown, escrita por Oh Jung-hee en 1979, en la que se muestra una visión de los acontecimientos de la época desde la perspectiva de los habitantes locales. En contraposición al texto anterior, en esta obra se refleja la creciente escalada de violencia que las prostitutas sufrieron a manos de los militares americanos. Con base en el análisis crítico del discurso, cotejo estos dos textos para revelar cómo determinados mecanismos lingüísticos contribuyen a la transmisión de una ideología patriarcal e imperialista.

Recepción: 19 de enero de 2020. / Aceptación: 3 de septiembre de 2020.

D.R. ${ }^{\circ}$ 2021. Estudios de Asia y África Licencia Creative Commons Atribución-NoComercial-SinDerivar (CC BY-NC-ND) 4.0 Internacional 
Palabras clave: otredad de las mujeres; prostitutas para militares; wianbu en la Corea del Sur de la posguerra; Chinatown de Oh Junghee; informe policial de Yongsan.

Abstract: This article compares two texts that offer different perspectives on the same situation of oppression imposed upon women by power. The analysis begins by examining an official report by the Seoul's Yongsan District police in 1971 that contains rhetorical guidelines to condone the crimes against Korean prostitutes committed by American soldiers, and to force the Korean people to treat the soldiers favorably. In parallel, the article focuses on Oh Jung-hee's Chinatown, a short story written in 1979. This work reveals how local residents perceived prostitutes at that time. In contrast to the previous text, Chinatown mainly describes how American troops were increasingly violent toward these women. A critical discourse analysis of these two texts reveals the use of linguistic devices to spread imperial and patriarchal ideology.

Keywords: otherness of women; comfort women; wianbu in post-war South Korea; Chinatown by Oh Jung-hee; Yongsan police report.

\section{Introducción}

A lo largo de la historia, los pueblos vencedores de los conflictos entre estados casi siempre se han atribuido el derecho a subyugar, esclavizar e, incluso, matar a los vencidos. En el caso de las mujeres, además, con frecuencia han sido utilizadas como moneda de cambio para compensar al vencedor. Así, en la península de Corea, más de 200000 mujeres, a través de engaños o por medio de la violencia, fueron sometidas a esclavitud sexual en centros militares japoneses durante el periodo de colonización iniciado en 1910 y que finalizó con la derrota de Japón en la Segunda Guerra Mundial (Chaure 2018, 170; Soh 2008, 2; Hynes 2004, 437). Esas mujeres son conocidas en coreano como wianbu: wian (consuelo) + bu (mujer). Ésa fue 
precisamente una de las consecuencias más atroces del colonialismo: la institucionalización y comercialización de las llamadas wianbu (Lie 1995, 311), la mayoría de las cuales no pudo nunca reinsertarse en la sociedad y acabó recluida en sus hogares, e incluso atentando contra su propia vida (Chaure 2018, 170).

La historia de sometimiento de las wianbu no acabó ahí. Al final de la Segunda Guerra Mundial, Corea del Sur se alió con las fuerzas armadas de las Naciones Unidas, encabezadas por Estados Unidos. Esto propició que se establecieran en el territorio bases militares americanas, en torno a las cuales emergieron zonas comerciales conocidas como gijichon. ${ }^{1} \mathrm{El}$ pueblo surcoreano padecía una gran crisis económica y numerosas mujeres se vieron obligadas a prostituirse en esas áreas para sacar adelante a sus familias (Lee 2013, 25; Park 2013, 80; Park 2015, 10). De esta forma, la prostitución dirigida a los extranjeros siguió expandiéndose (Lie 1995, 310). Incluso los japoneses practicaban el turismo sexual con el visto bueno del régimen coreano (Park 2013, 75), que lo consideraba un medio para obtener divisas para el país (13).

Este trabajo de investigación se centra en los acontecimientos acaecidos en la década de 1970 en los gijichon asentados en Corea del Sur, cuando el gobierno surcoreano, por su debilitamiento, buscaba la alianza con Estados Unidos y, para lograrlo, se sirvió de las wianbu y de su valor como objetos. Para ello, analizo el informe emitido por la policía del distrito de Yongsan de Seúl en 1971. En este comunicado, con un lenguaje retórico, se enmascaraba y justificaba la dureza de la conducta de los militares americanos con las wianbu, al mismo tiempo que se imponía a la población coreana un trato benévolo, complaciente y servicial a esos soldados. En contraposición, examino también la novela Chinatown, ${ }^{2}$ escrita por Oh Junghee en 1979, obra testimonial que evidencia la percepción de

${ }^{1}$ Gijichon son áreas circunscritas a las zonas militares americanas donde se encuentran viviendas y locales en los que se ejerce la prostitución.

${ }^{2}$ El libro coreano fue traducido al inglés con el título de Chinatown [el barrio chino]. 
los habitantes locales sobre las wianbu y muestra la creciente escalada de violencia sufrida por ellas a manos de militares americanos que actuaban impunemente con el beneplácito del régimen dictatorial. Ambos textos, pese a que comparten referentes temporales y espaciales, muestran visiones antagónicas de una misma realidad, lo que alerta acerca de la necesidad de reflexionar en profundidad sobre la violencia y el maltrato hacia las mujeres y sobre cómo la sociedad los ha interpretado.

\section{Marco teórico}

El objetivo de esta investigación es mostrar cómo los textos seleccionados representan el mundo de forma sesgada y opuesta. Para ello, aplico como marco teórico el análisis crítico del discurso surgido de los estudios de lingüistas como Fairclough $(1992,2012)$. El modelo se basa en el principio de que los textos nunca pueden ser entendidos ni analizados de forma aislada, sino sólo en relación con el contexto discursivo y social. En otras palabras, nuestra sociedad conforma los textos y, a su vez, los textos influyen en la propia sociedad. Fairclough $(1992,4)$ trata de hacer evidentes las implicaciones ideológicas y sociales del uso del lenguaje teniendo en cuenta el concepto de discurso y su análisis tridimensional: i) el lenguaje, ii) la práctica del discurso y sus procesos de producción e interpretación, y iii) la práctica sociopolítica y cultural. A esta teoría, Van Dijk (1998, 162) aporta el concepto del poder en los contextos sociales. Se centra en el papel del discurso en una situación de abuso de poder o de dominación entre grupos sociales: ciertos grupos tienen poder porque son capaces de controlar los actos y los pensamientos de otros menos poderosos o sin poder.

Para tratar esa dicotomía entre grupos, Al-Saidi $(2014,96)$ recurre al concepto de otredad, entendido como la división entre uno mismo y otro, colonizador y colonizado, poderoso e impotente, expresada a través del lenguaje como fuente de reproducción de la desigualdad en la sociedad. Esta oposición 
presupone que el grupo de poder tiene acceso privilegiado y autorizado a los escasos recursos sociales y políticos, como la información, el conocimiento, el gobierno o los medios de comunicación de masas.

A diferencia de otras teorías sociales y culturales, el análisis crítico del discurso se basa en las interpretaciones lingüísticas de los textos, pero al mismo tiempo se distingue de los modelos puramente lingüísticos en su enfoque sociopolítico. Es decir, cualquier elección lingüística y temática puede hacer que el discurso se convierta en herramienta de persuasión o propagación ideológica. Si el ciclo se repite, un discurso llega a moldear y remodelar la realidad y viceversa (Fairclough 2012, 457). Por tanto, se trata de una perspectiva interdisciplinar en la que es posible combinar el análisis textual y social y revelar el uso de mecanismos lingüísticos manipulativos que, en este caso, conducen a la transmisión de la ideología patriarcal e imperialista.

Un aspecto particularmente relevante para el análisis crítico del discurso es "el análisis de varias formas de significados implícitos o indirectos, como implicaciones, presuposiciones, alusiones, vaguedades, etc.", esto se debe a que "los significados implícitos están relacionados con las creencias subyacentes, pero no se afirman de manera abierta, directa, completa o precisa" (Van Dijk 2001, 104). Por ende, se requiere un análisis de contenido que incluye la selección de temas, proposiciones y elementos léxicos, porque tales parámetros parecen estar más directamente relacionados con la actitud y la ideología sexistas (99). Más específicamente, en un discurso se pueden distinguir las estructuras globales y las locales. El análisis tópico o macroestructural trata de descubrir la información más relevante de un texto (102). En un nivel micro, los significados locales abarcan el significado de las palabras, las estructuras de las proposiciones, la coherencia y otras relaciones entre proposiciones (103), con los que se expresaría la otredad del grupo sin poder. De este modo, este estudio intenta dar visibilidad a aquellas mujeres tratadas como "otras" frente a los agentes de poder y desvelar su punto de vista, omitido con frecuencia, 
a través del análisis de los significados implícitos de los dos textos elegidos.

\section{Antecedentes históricos de las directrices de la policía del distrito de Yongsan}

Para aportar una visión más completa, recurro al contexto sociopolítico de la época, esencial para interpretar el documento con las directrices de la policía del distrito de Yongsan. El dictador coreano Park Chung-hee llegó al poder tras un golpe militar en 1961 y gobernó hasta su asesinato en 1979. Desde el comienzo de su régimen, favoreció un crecimiento económico basado en las tradiciones culturales coreanas, conservadas a través del neoconfucianismo, en las que se enfatizaban la lealtad, el patriotismo y la cooperación. Pese a ello, una parte importante de los beneficios económicos se obtenía de actividades antagónicas a esos valores, como la prostitución, que se expandió y se consolidó en las décadas de 1960 y 1970 (Park 2015, 13; Lee 2018, 146). Como la prostitución en Corea del Sur no estaba legalizada, sino simplemente tolerada, J.-M. Park $(2013,78)$ se refiere al sistema como el "régimen de la regulación-tolerancia”, en oposición a un "régimen de regulación-autorización”, ya que este último implicaría la legalización de la actividad.

Durante ese periodo, las instalaciones de los gijichon fueron una fuente de divisas importante para la economía y el desarrollo del país. En ellas, el gobierno surcoreano consintió y fomentó el ejercicio de la prostitución para mejorar las relaciones con Estados Unidos y restaurar la economía del país tras la posguerra (Moon 1997; Lee 2007; Kim 2015; Park 2015; Lee 2018). En la provincia de Gyonggi-do, ${ }^{3}$ por ejemplo, las aproximadamente 4000 wianbu generaron ocho millones de dólares en 1970, al mismo tiempo que cada una de ellas mantenía a un promedio de cuatro familiares (Lee 2006, 128). Según las estimaciones de J. Kim $(2017,20)$, 25\% del PNB de Corea

${ }^{3}$ Gyonggi-do es una provincia que rodea la provincia de Seúl. 
del Sur en la década de 1960 se originó gracias a las relaciones con los americanos, con 46000 coreanos ganando 70 millones de dólares solo en 1969.

En 1969, casi a la mitad de su mandato, Park Chung-hee tuvo que hacer frente a la doctrina Nixon, que implicaba la retirada del apoyo militar de Estados Unidos a sus aliados asiáticos (León Manríquez y López Aymes 2009, 156). El siguiente extracto del periódico coreano JoonAng Ilbo refleja cómo los militares americanos fueron el principal aporte a la economía local de la época y cuáles fueron las consecuencias negativas de su repliegue:

Con el rumor de la retirada del Ejército de Estados Unidos, los soldados poco a poco dejaron de frecuentar los alrededores de los gijichon, que económicamente dependían de ellos. La recesión comenzó a hacerse patente: se devaluó el precio de los bienes inmuebles y se produjeron altas tasas de desempleo entre la población nativa, todo ello como consecuencia del cierre de varios establecimientos de entretenimiento para adultos (Kim 1970, 7). ${ }^{4}$

El daño económico que podría provocar la reducción de tropas sería significativo para Corea del Sur. Ante la amenaza inminente de un vacío de seguridad nacional y de falta de fondos económicos, el gobierno coreano intentó contrarrestar la propuesta de retirada mediante estrategias que favorecieran una mayor cooperación con Estados Unidos. Finalmente, las alianzas no se rompieron, entre otros motivos, por el importante papel que tuvo la normalización del indulto al uso de la prostitución por parte de los soldados americanos (Park 2015, 3; Kim 2015, 154).

Este requerimiento de Estados Unidos sirvió para enmascarar altercados y crímenes violentos de los soldados estadounidenses contra la población civil surcoreana, así como conflictos surgidos entre los militares blancos y sus compañeros negros (Park 2015, 9; Lee 2006, 133; Kim 2015, 155-156). A continuación, en el cuadro 1 , se pueden ver algunos titulares

${ }^{4}$ La traducción del artículo del coreano al español es mía. 
de noticias que seleccioné de dos de los principales periódicos coreanos, Chosun Ilbo y JoongAng Ilbo, del periodo entre 1968 y 1972, en los que se evidencia la violencia ejercida por los soldados en contra de las wianbu, así como la impunidad de la que gozaron.

\section{Cuadro 1. Titulares de periódicos coreanos desde 1968 hasta 1972*}

\begin{tabular}{|c|c|c|c|}
\hline$A \tilde{n} o$ & Fecha & Periódico & Traducción al español \\
\hline \multirow[t]{6}{*}{1968} & 27 de abril & Chosun Ilbo & $\begin{array}{l}\text { Otro incendio provocado } \\
\text { por un soldado } \\
\text { estadounidense acaba } \\
\text { con la vida de un niño } \\
\text { en la casa de una wianbu. }{ }^{\text {I }}\end{array}$ \\
\hline & 2 de mayo & Chosun Ilbo & $\begin{array}{l}\text { Un soldado estadounidense } \\
\text { asesina a una reianbu } \\
\text { y apela al tribunal de su país } \\
\text { acusando al tribunal coreano } \\
\text { de "injusto". .I }\end{array}$ \\
\hline & 14 de mayo & JoongAng Ilbo & $\begin{array}{l}\text { El incendio provocado } \\
\text { en la casa de una wianbu } \\
\text { causa su muerte. }{ }^{\text {III }}\end{array}$ \\
\hline & 7 de septiembre & Chosun Ilbo & $\begin{array}{l}\text { Un soldado estadounidense } \\
\text { destinado en Corea fusila } \\
\text { a una wianbu. } .^{\mathrm{IV}}\end{array}$ \\
\hline & 17 de septiembre & JoongAng Ilbo & La asesinada es una rwianbu. ${ }^{v}$ \\
\hline & 23 de octubre & Chosun Ilbo & $\begin{array}{l}\text { Tras la ausencia de } \\
\text { cooperación de EE.UU. } \\
\text { en la investigación a uno } \\
\text { de sus soldados, fiscales } \\
\text { coreanos ordenan } \\
\text { la instrucción del caso } \\
\text { del asesinato de la wianbu. }{ }^{\mathrm{VI}}\end{array}$ \\
\hline 1969 & 7 de enero & Chosun Ilbo & $\begin{array}{l}\text { Unos diez soldados } \\
\text { estadounidenses secuestraron } \\
\text { y violaron en grupo a una } \\
\text { wianbu dentro del cuartel. }\end{array}$ \\
\hline
\end{tabular}




\section{CUADRO 1. (continuación)}

\begin{tabular}{|c|c|c|c|}
\hline & 7 de enero & JoongAng Ilbo & $\begin{array}{l}\text { Militares negros con } \\
\text { conducta violenta. }^{\text {VIII }}\end{array}$ \\
\hline & 14 de enero & Chosun Ilbo & $\begin{array}{l}\text { Wianbu muere estrangulada. } \\
\text { El soldado estadounidense, } \\
\text { pese a mostrar resistencia, } \\
\text { fue arrestado. Ejerce su } \\
\text { derecho a guardar silencio. IX }\end{array}$ \\
\hline & 14 de enero & Chosun Ilbo & $\begin{array}{l}\text { En el caso del asesinato } \\
\text { de la wianbu: veredicto } \\
\text { de inocencia para el cabo } \\
\text { tercero Smallwood. }{ }^{\mathrm{X}}\end{array}$ \\
\hline & 20 de mayo & Chosun Ilbo & $\begin{array}{l}\text { Soldado estadounidense } \\
\text { asesina a una wianbu. }{ }^{\text {XI }}\end{array}$ \\
\hline & 22 de agosto & JoongAng Ilbo & $\begin{array}{l}\text { Soldado estadounidense } \\
\text { provoca un incendio. XII }\end{array}$ \\
\hline & 31 de octubre & Chosun Ilbo & $\begin{array}{l}\text { El caso de asesinato de una } \\
\text { wianbu. Sentencia de diez } \\
\text { años de cárcel para el soldado } \\
\text { estadounidense. XIII }\end{array}$ \\
\hline 1970 & 6 de noviembre & JoongAng Ilbo & $\begin{array}{l}\text { Wianbu que servía al ejército } \\
\text { de EE.UU. aparece muerta } \\
\text { completamente desnuda. XIV }\end{array}$ \\
\hline 1971 & 19 de febrero & JoongAng Ilbo & $\begin{array}{l}\text { El rechazo de una wianbu } \\
\text { a la convivencia termina } \\
\text { con gas lacrimógeno arrojado } \\
\text { a su casa. }{ }^{X v}\end{array}$ \\
\hline & 4 de mayo & JoongAng Ilbo & Protesta de las wianbu. XVI \\
\hline & 17 de julio & Chosun Ilbo & $\begin{array}{l}\text { Tras acostarse con un militar } \\
\text { de EE.UU., la wianbu es } \\
\text { asesinada.XVII }\end{array}$ \\
\hline 1972 & 8 de marzo & Chosun Ilbo & $\begin{array}{l}\text { Bupyeong, el juicio original } \\
\text { del asesinato de la wianbu } \\
\text { es anulado y el militar } \\
\text { de EE.UU. es declarado } \\
\text { inocente. XvIII }\end{array}$ \\
\hline
\end{tabular}

* La traducción de los titulares de periódicos del coreano al español es mía. 


\section{CuADRo 1. (conclusión)}

I https://newslibrary.chosun.com/view/article_view. html?id=1446919680427m10313

II https://news library.chosun.com/view/article_view. html?id=1447319680502m10718

III https://news.joins.com/article/1160510

IV https://newslibrary.chosun.com/view/article_view. html?id=1458319680907m1036

${ }^{v}$ https://news.joins.com/article/1173729

vi https://newslibrary.chosun.com/view/article_view. html?id=1462219681023m1035

vil https://news library.chosun.com/view/article_view. html?id=1468419690107m10719

VIII https://news.joins.com/article/1184081

IX https://newslibrary.chosun.com/view/article_view. html?id=1469019690114m1072

x https://newslibrary.chosun.com/view/article_view. html?id=1469019690114m10715

XI https://newslibrary.chosun.com/view/article_view. html?id=1479719690520m10720

XII https://news.joins.com/article/1207611

XiII https://newslibrary.chosun.com/view/article_view. html?id=1493819691031m1039

XIV https://news.joins.com/article/1263795

Xv https://news.joins.com/article/1279044

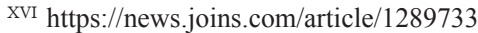

Xvil https://newslibrary.chosun.com/view/article_view. html?id=1546619710717m10719

xvir https://newslibrary.chosun.com/view/article_view. html?id=1566519720308m1078

Cuando Park Chung-hee tomó el poder en 1961, la prensa cayó bajo una férrea censura que acabó con la mayor parte de los periódicos; sólo 34 sobrevivieron a duras penas (Ríos 2000, 76). JoongAng Ilbo era uno de los periódicos comerciales más representativos de los años setenta, y Chosun Ilbo era considerado el periódico con mayor trayectoria, pero con el menor número de periodistas mujeres empleadas en toda su historia (Choi 2015, 294). Es decir, la situación llegó a ser tan grave que incluso las publicaciones conservadoras se vieron obligadas a cubrir la violencia que sufrían las wianbu. 
En ese contexto, algunos distritos se caracterizaron por ser especialmente condescendientes con los conflictos producidos por los militares estadounidenses. Entre ellos Yongsan, distrito de Seúl, cuyo jefe de policía emitió un documento oficial el 14 de julio de 1971 dirigido a la población local que trabajaba en la zona del gijichon. Ese documento, que revela las políticas del gobierno para controlar a las wianbu, recogía las normas que debían seguir las prostitutas que prestaban sus servicios a los soldados americanos. Hay que destacar que ese tipo de estrategias no solían ser explícitas y que, por ello, el documento, que es de los pocos que se han conservado, tiene un alto valor testimonial.

\section{Chinatown de Oh Jung-hee}

El segundo texto, Chinatown, es una novela corta escrita en 1979 por Oh Jung-hee (1947-) cuya trascendencia hace que en la actualidad forme parte del currículo en los planes de estudio de las asignaturas de lengua y literatura coreanas de educación secundaria. La autora, una de las escritoras surcoreanas más reconocidas del siglo xx, nació en Seúl el 9 de noviembre de 1947, tres años antes del estallido de la Guerra de Corea. Su familia, originaria de Haeju, en Corea del Norte, emigró a Corea del Sur, donde vivieron de forma austera como refugiados. Pese a ello, Oh Jung-hee se licenció en escritura creativa en 1968 por la Escuela de Arte Seorabeol de Seúl.

Su trabajo literario se caracteriza por reflejar el ámbito familiar desde la perspectiva de la mujer. La obra que nos ocupa está íntimamente relacionada con su experiencia vital: Chinatown es un barrio ubicado en Incheon, una ciudad portuaria de la costa oeste cercana a Seúl y puerta de entrada para las tropas estadounidenses a la zona. La autora y su familia se asentaron en ese barrio humilde de inmigrantes que en la actualidad es uno de los lugares turísticos más populares de Corea del Sur. 
La historia se narra desde la perspectiva de una niña anónima de nueve años cuya familia se refugia en las afueras de Incheon, rodeada por campamentos militares estadounidenses y escombros de la posguerra. La vida de la narradora está marcada por la pobreza, el sexo y la muerte. Los niños se ganan la vida robando el carbón de los trenes y el trigo de los molinos de harina. Las mujeres se ven empujadas a la prostitución para paliar el hambre y otras necesidades de sus familias. La mejor amiga de la niña, Chi-ok, se siente atraída por el glamour, el exotismo y el lujo de una realidad difuminada que representa su vecina, una prostituta llamada Maggie. Sin embargo, el desenlace de su vida desenmascara una historia trágica muy diferente a la fantasía descrita por Chi-ok: Maggie muere violentamente a manos de su amante militar afroamericano.

El texto tiene gran valor tanto por su calidad literaria como por la valentía de la autora al tratar un tema tan comprometedor. Debido a la censura, escribir una novela fue una manera de revelar la realidad sufrida por las mujeres bajo el régimen autoritario y patriarcal. Además, conocer esa realidad desde la perspectiva íntima y personal que ofrece la autora es fundamental para comprender y empatizar con el dolor de aquellas mujeres identificadas como "otras".

\section{Antecedentes de los estudios sobre la otredad de las mujeres}

Partiendo del concepto otredad, S. I. Lee (2007, 119-120) explica que el confucianismo refuerza la autoridad de los hombres y coloca a las mujeres en una posición periférica. Como resultado, las mujeres se identifican a sí mismas como "otras". En esa relación, además, se da la circunstancia de que el grupo de poder llega a ser sustituido por otro aún más poderoso: tras la expulsión oficial de los colonos japoneses de la península de Corea, ésta se vio sometida a un nuevo "colonizador", el imperialismo cultural de Estados Unidos (206), que puso de manifiesto una 
profunda admiración a la superioridad yanqui por parte de la población coreana. Lim $(2017,243)$ señala que, en las décadas de 1950 y 1960, el pueblo coreano anhelaba el desarrollo y la civilización avanzada que simbolizaban los estadounidenses. Como consecuencia, los soldados acabaron convirtiéndose en agentes protagonistas en Corea del Sur, mientras que los coreanos eran considerados "otros" en su propio país. En la época poscolonial fue frecuente ese dominio cultural de los países occidentales sobre muchos países asiáticos. En ese contexto, las mujeres eran consideradas seres vulnerables e ingenuos, especialmente susceptibles a la influencia y la manipulación de los deseos imperialistas.

N. Y. Lee $(2008,95-97)$ se centra en las wianbu surcoreanas que sirvieron al ejército estadounidense. Esta autora argumenta que la identidad de las prostitutas se define en oposición al resto de las mujeres. Mientras a las virtuosas se les encuadra en un estándar que les confiere superioridad moral, a las prostitutas se les rechaza por no cumplir el código de conducta para ser un miembro legítimo de la nación. Sin embargo, en la década de 1970 esas mismas mujeres se convirtieron en un pilar fundamental para la recuperación económica de la nación. Esto derivó en que, al tiempo que eran repudiadas, eran elogiadas como una suerte de heroínas del país. El estudio histórico de J.-M. Park $(2013,80)$ revela que el gobierno, en efecto, favoreció la prostitución como una forma de recuperación económica, pese a lo cual marcó los límites de la "comunidad decente" mediante jerarquías entre las mujeres castas y las caídas, así como entre hombres y mujeres.

En ese contexto surgió una nueva humillación: la inquietud del gobierno coreano de controlar las enfermedades venéreas que transmitían las prostitutas. J.-M. Park $(2015,25)$ aclara que la principal preocupación era evitar el contagio y preservar la salud de los soldados americanos. El régimen autoritario garantizó tácitamente el privilegio sexual de los ciudadanos varones y las prostitutas se convirtieron en no ciudadanas. La Ley de Prevención de Enfermedades Infecciosas permitió a 
las autoridades controlar la salud de las prostitutas incluso obviando algunos derechos básicos garantizados por la Constitución, hasta el punto de que podían internarlas en contra de su voluntad en el centro de detención conocido como Monkey House. $^{5}$

No sólo las wianbu eran consideradas personas marginales y rechazadas, como una suerte de "otros", sino también sus hijos mestizos. Hwang $(2015,191)$ pone de manifiesto la visión de la niña ante los prejuicios contra la prostituta coreana Maggie y su hija mestiza Jenny. Según J. H. Park (2016, 241242), son ignorados por la población local y sus instituciones. De ahí surge el concepto de "diáspora coreana", que no hace referencia a una dispersión fuera del país, pues nunca salieron de él, sino a la falta de integración a la sociedad. La diáspora está representada en fotografías en las que los ojos de los niños transmiten una sensación de temor y ansiedad, mientras que la postura y la expresión facial de las mujeres carecen de emoción y muestran un gesto incómodo como signo pasivo de rechazo a los soldados. En ese esquema de dicotomías -estadounidenses frente a coreanos, hombres frente a mujeres, castas frente a prostitutas-, las wianbu ocupan el escalafón más bajo y representan al grupo social sin ningún tipo de poder.

\section{Antecedentes de los estudios sobre Chinatown}

Chinatown ha sido objeto de múltiples estudios. En algunos se hace hincapié en el papel de la joven narradora, de la que ni siquiera sabemos su nombre, y en cómo cuenta la vida de los que la rodean poniendo de relieve la perspectiva de la otredad. Y.-H. Oh $(2007,275)$ habla sobre la discriminación de los refugiados de guerra $\mathrm{y}$, por ende, de la identidad ambigua de la

${ }^{5}$ Las wianbu que resultaron infectadas o sospechosas de enfermedades venéreas fueron enviadas al centro de detención Monkey House. En ese lugar, en el que hasta las ventanas tenían rejas, se les inyectaban altas dosis de penicilina y se les mantenía presas hasta que estuvieran "curadas". 
narradora. La joven no ha establecido su identidad como una persona independiente, sino como una mujer dominada por el patriarcado (Jang 2006, 322). Esto se debe a que vive en un país en el que los hombres acaparan el rol activo: durante la guerra fueron los encargados de ejercer la violencia y después, una vez que el conflicto acabó, tuvieron en sus manos la reconstrucción del país. En el lado opuesto, la existencia de las mujeres está restringida al ámbito doméstico.

A pesar de que la batalla bélica había terminado, para las mujeres de Chinatown los efectos de la guerra persisten porque siguen viviendo en una sociedad patriarcal opresiva en la que se les identifica como las “otras” (Jeong 2006, 203). Asimismo, las mujeres en Chinatown tienen una responsabilidad dentro de la casa, porque la sociedad las obliga a asumir el papel de "madres sabias" y "buenas esposas" (Lee 2018, 145). De esta forma, la mujer se limita a ser un sujeto reproductivo y un objeto sexual. En ese ambiente, la niña, que ha sido testigo de la vida de su madre, reniega de la maternidad como única vía existencial. Esa negación puede observarse en el miedo que manifiesta a que la madre pueda fallecer en alguno de sus muchos alumbramientos. Guahk $(2018,327-238)$ habla de la paradójica situación que nos presenta la narradora: anhela el amor de su madre mientras rechaza ser como ella por estar constantemente embarazada. Tal parece que todas las mujeres de la novela están destinadas a un final trágico. J. Kim (2017, 93) centra la atención en esa relación entre la mujer y la muerte, que se observa en el fin de su abuela y el asesinato de su vecina Maggie.

Por otro lado, hay estudios que se centran en el valor simbólico de Chinatown como espacio: se trataría de una reminiscencia del imperio chino que dominó durante siglos la península coreana. En el tiempo en el que se sitúa la novela, los lazos entre Corea y China habían quedado prácticamente rotos con la invasión colonial japonesa y la posterior presencia de Estados Unidos. En ese contexto, el barrio chino se convirtió en un reducto que dio rienda suelta al rechazo del pueblo 
coreano hacia los chinos residentes. Y.-H. Oh $(2012,225)$ configura la ciudad portuaria de Incheon como una geografía literaria que despierta los traumas de la guerra. Por su parte, H. S. Lee $(2008,73)$ centra su atención en la singularidad de Chinatown como un lugar desconocido para la narradora y en la aceptación de su otredad como identidad aislada en su comunidad. Joo $(2016,77)$ divide topográficamente las dos realidades: Incheon y Chinatown. La primera representa la violencia y el capitalismo traído por los soldados estadounidenses a Corea del Sur, mientras la segunda es un símbolo del patriarcado y el chovinismo en virtud del cual las mujeres son explotadas.

En este artículo aúno las teorías expuestas y aplico el análisis crítico del discurso para estudiar los significados tanto explícitos como implícitos. De este modo, pretendo dar visibilidad a la perspectiva de las "otras", de las mujeres oprimidas por los agentes de poder, cuya voz está omitida en los dos textos seleccionados.

\section{Vulnerabilidad de las mujeres frente al poder}

Inicio el análisis enfocándome en la representación de los grupos sociales poderosos en contraste con las wianbu. En el documento histórico oficial de la policía de Yongsan se emplean diferentes recursos lingüísticos para dicha representación:

Aunque se confía en que hasta ahora han brindado su mejor servicio al ejército estadounidense, se han causado molestias involuntariamente a algunos soldados. Debemos reflexionar sobre esas cosas del pasado y corregirlas. Deben saber que incluso esos incidentes triviales son aprovechados por el enemigo. En estas circunstancias, también deben saber que están ayudando inconscientemente a los enemigos de la patria, debilitando la seguridad nacional. Deben comprender que el ejército estadounidense se encuentra en Corea del Sur para ayudarles a defender la nación de la invasión y las actividades bélicas de Corea del Norte (Policía del distrito de Yongsan 1971, 1). 
Figura 1. Directrices de la policía del distrito de Yongsan

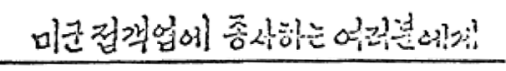

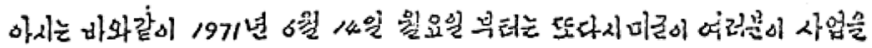
하시르 지역에 마음대로 드나둘수 했게디었읍시다.

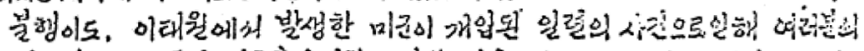

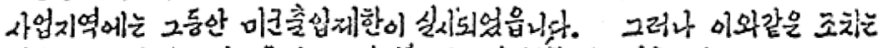

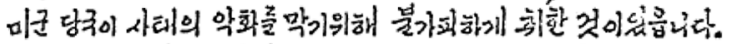

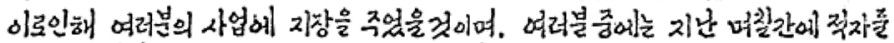

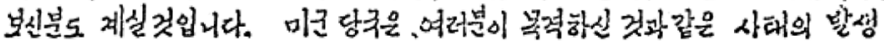

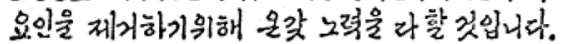

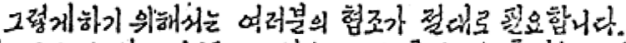

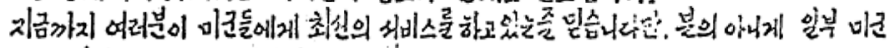

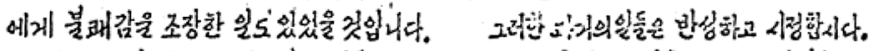

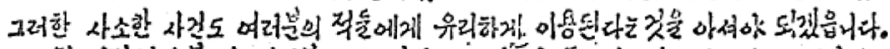

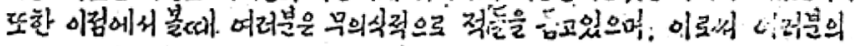

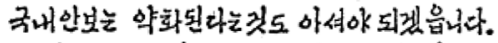

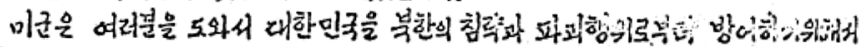
한극에 와 있다늘 갯을 아삽시오.

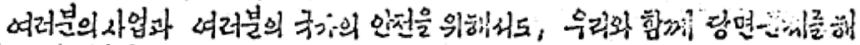

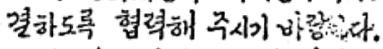

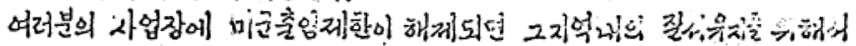

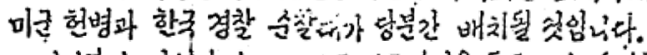

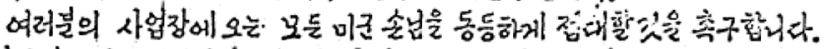

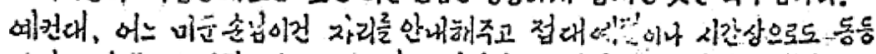

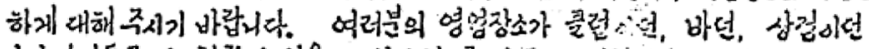

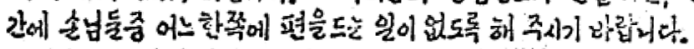

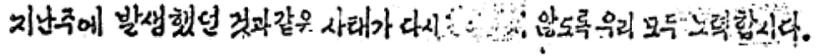

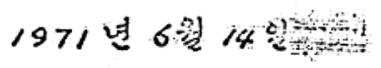

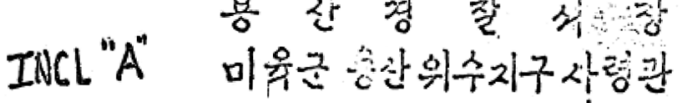

Fuente: Policía del distrito de Yongsan, 1971.

Este fragmento redactado de manera impersonal muestra varios sujetos: Estados Unidos aparece como el protector del país, mientras que Corea del Norte es lexicalizada como el ene- 
migo. Por su parte, las autoridades coreanas aparecen en tercer plano, difuminadas, sin asumir ninguna responsabilidad en los incidentes ni en sus posibles soluciones. La ausencia del gobierno surcoreano como agente visible y la imposición unilateral de regulaciones a las mujeres coreanas en beneficio del ejército americano muestran el neoimperialismo estadounidense y la inamovible jerarquía.

Se acusa directa y únicamente a las mujeres de ser causantes y culpables de dichos incidentes. A lo largo del informe, la ambigüedad prevalece mediante la elección léxica para aludir a las wianbu: se recurre a expresiones eufemísticas o confusas al indicar que las prostitutas "han brindado su mejor servicio", y al mismo tiempo se leen frases como "han causado molestias involuntariamente a algunos soldados". Asimismo, se usan otras expresiones poco precisas para ocultar o minimizar los crímenes cometidos por los militares: "esas cosas del pasado" $\mathrm{y}$ "el incidente trivial".

Otro rasgo lingǘstico que destaca es el frecuente uso de la modalidad, estrategia empleada para enunciar la necesidad o la posibilidad de acciones y que se puede expresar mediante diversas realizaciones lingüísticas, como complementos modales, adjetivos, verbos léxicos o estructuras condicionales. Hay dos tipos básicos de modalidad: la epistémica, que refleja un grado de certeza relativamente bajo, y la deóntica, que connota obligación, poder, autoridad, deber e insistencia. El cuadro 2 muestra que en el documento policial predomina la modalidad deóntica, como prueba de la opresión del gobierno coreano a estas mujeres. A través de la repetición y la variación de enunciados modales de alto grado impositivo se observa la dinámica unilateral de poder impuesto sobre el destinatario.

Toda esa violencia también aparece en Chinatown y es particularmente incuestionable cuando la narradora habla de la tragedia sufrida por su vecina Maggie, asesinada por su pareja, un soldado afroamericano con el que convivía. Cuando el cuerpo de la prostituta, que ha sido arrojado por el balcón, aún 
CuAdro 2. Modalidad deóntica y epistémica

\begin{tabular}{cll}
\hline$N$ & Modalidad deóntica & Modalidad epistémica \\
\hline 1 & Urge & Se convertirá \\
2 & Reflexionemos & Estará \\
3 & Corrijamos & Hará todo \\
4 & Tendrán que saber & Habría estado \\
5 & Tendrán que comprender & \\
6 & Deben saber & \\
7 & Deben comprender & \\
8 & Se espera que cooperen & \\
9 & Se espera que traten & \\
10 & Se espera que no haya & \\
11 & Hagamos esfuerzos & \\
12 & Inevitablemente & \\
13 & Absolutamente necesario & \\
\hline
\end{tabular}

Fuente: elaboración propia.

yace en el suelo, las fuerzas del ejército estadounidense acuden rápidamente a socorrer al asesino para alejarlo del lugar de los hechos. Mientras toda la ciudad observa con compasión el cuerpo sin vida tirado en la calle, el asesino se marcha del lugar, ebrio, entre risas. Esto refleja tanto la impunidad neoimperial de Estados Unidos como la impotencia servil de Corea del Sur ante la agresión machista. Además, la conducta del asesino revela una realidad en la que las autoridades locales pocas veces hacían justicia en casos como éste, como se vio en los titulares de los periódicos analizados. El siguiente fragmento sirve como testimonio de la marginalidad que sufrían las wianbu en la configuración social de la otredad:

Más tarde, un camión militar estadounidense apareció entre sirenas de emergencia, abriéndose paso y dividiendo el tumulto en dos. Bajo los 
faros delanteros, Maggie yacía en el suelo. Su cabello teñido, largo y voluminoso la envolvía como un halo. Alguien susurraba que la habían tirado desde el balcón.

El negro todavía estaba borracho y desnudo. Un soldado lo cubrió con el uniforme. Lo subieron al camión con la chaqueta aún desabrochada, riéndose mientras se alejaba (Oh 2002, 238).

En el fragmento se alude al asesino de forma impersonal con la expresión "la habían tirado desde el balcón", pese a que todos sabían quién había sido el responsable. De nuevo, la población local se limita a ser un testigo silencioso y pasivo que se aparta ante la llegada de los soldados. En cambio, éstos actúan con determinación, pero sólo para protegerse entre ellos, no para defender a la víctima. Pese a todo, la novela intenta dignificar y divinizar a la prostituta al indicar que su pelo "la envolvía como un halo". De esta forma, su imagen contrasta con la del soldado, convertido en un fantoche incapaz de controlar su propia conducta y que debe ser movido por sus propios compañeros, que "lo subieron al camión".

Estos fragmentos ponen de manifiesto dos posturas contrapuestas: el informe policial muestra el favoritismo hacia Estados Unidos; Chinatown revela la atrocidad del soldado estadounidense. Mientras que la novela, un texto ficcional, pretende ser reflejo de la realidad, el informe policial, pese a ser un documento real, intenta enmascararla.

\section{Yangglbo en contraposición al negocio de la hospitalidad}

En los medios de comunicación surcoreanos, para nombrar a las prostitutas que sirven a los miembros del ejército estadounidense se utilizan los eufemismos wianbu [mujer de consuelo] o migun wianbu [mujer de consuelo para el ejército de Estados Unidos]. Sin embargo, ninguno de los dos términos aparece en los textos aquí analizados. En el informe de la policía de Yongsan no se encuentra mención explícita alguna a la prostitución. Para referirse a las prostitutas se recurre 
a un forzado circunloquio con el fin de manipular el mensaje: "A ustedes, que se encuentran prestando el servicio en pro del compromiso con el negocio de la hospitalidad para el ejército de Estados Unidos":

a) El pronombre personal de cortesía para la segunda persona del plural, "ustedes", tiene dos implicaciones. Primero, el uso formal muestra el respeto al destinatario. En segundo lugar, pone distancia entre el hablante y el destinatario. Además, se repite 17 veces a lo largo del documento, lo que refuerza esos efectos.

b) Frente a verbos habituales como trabajar o vender, "se encuentran prestando el servicio en pro del compromiso" es una expresión eufemística y artificial que glorifica la prostitución.

c) La elección léxica "el negocio de la hospitalidad" no remite de ninguna manera a la prostitución o al negocio del sexo, y no está documentada en ningún otro texto con ese valor. Este ambiguo concepto no recoge de forma clara a quién se dirige el documento, aunque resulte obvio. Todas estas frases y estos eufemismos pretenden enmascarar actitudes que entran en contradicción con los valores tradicionales.

El término wianbu tampoco se utiliza en Chinatown; tanto los adultos como los niños recurren a la palabra tabú yanggalbo [puta para yanquis]. Se trata de un epíteto insultante: abuelas y madres opinan que las yanggalbo son indecentes y obscenas porque se acuestan con hombres extranjeros, lo cual dista mucho del concepto tradicional de lo que es ser una mujer digna:

Mi abuela y mi madre le pusieron el nombre de "casa de yanggalbo" a la casa en la que vivía Chi-ok. Entre las casas de estilo japonés, la nuestra era la única de la calle en la que no se alquilaban habitaciones a las yanggalbo. Maggie dejaba las puertas abiertas y se precipitaba a los brazos de los soldados estadounidenses mientras ellos rodeaban su cintura. En 
su terraza colgaba prendas íntimas de varios colores y sus mantas sucias con la salvaje humedad de la noche anterior. La abuela, que consideraba que la ropa de las mujeres, y sobre todo la interior, debía secarse dentro de casa, al ver la escena en la vivienda de Chi-ok apartó la vista mientras las llamaba mangjong (Oh 2002, 226-227).

En el marco de la tradición cultural neoconfucianista y de las normas bajo las que se rige la moral conservadora de la sociedad coreana, las mujeres solteras que mantenían relaciones sexuales con extranjeros eran señaladas como seres contaminados y eran rechazadas porque se consideraba que traicionaban la pureza racial coreana. Por ello, la abuela llama mangjong a Maggie, expresión similar a la popular "garbanzo negro" u "oveja negra". La familia conservadora de la narradora se rige por un férreo concepto de la dignidad que quiere proteger, y por ese motivo no alquila habitaciones a ninguna yanggalbo, a pesar de que era una práctica común en el vecindario para ganar dinero. En contraste, nos encontramos ante el deseo sincero de la amiga íntima de la narradora, Chi-ok, la cual dice abiertamente que quiere convertirse en yanggalbo:

Cuando crezca, seré una yanggalbo. Maggie me dijo que me regalaría todas sus cosas, sus collares, sus zapatos y su ropa [...].

Voy a ser una yanggalbo (Oh 2002, 229).

Cuando salga, me convertiré en una yanggalbo (233).

A diferencia de los adultos, las niñas sueñan con crecer para tener vidas diferentes a las de sus madres. En el caso de Chi-ok, esa ensoñación de una nueva vida se manifiesta a través de su atracción por los productos extranjeros. Ella ve que su única posibilidad de salir de la pobreza es llegar a ser como Maggie. Para ella, Maggie es una figura admirable e inspiradora.

Como se observa, los dos textos muestran denominaciones contrapuestas que dejan en evidencia el modelo de otredad. El documento de la policía opta por una expresión forzada, 
ambigua y eufemística, que refleja que la autoridad local quería evitar la mención directa a las prostitutas y marcar una distancia formal. En cambio, en Chinatown se recurre a un término despectivo, vulgar e informal: yanggalbo. Todas estas elecciones léxicas son el reflejo, de nuevo, de dos posturas opuestas ante una misma realidad.

\section{Los mestizos abandonados}

Durante la guerra y la posguerra, fruto de las relaciones entre soldados americanos y mujeres locales, nacieron numerosos niños, aunque no se sabe exactamente cuántos, porque no existe registro alguno. A continuación, me detengo en el análisis de la figura de estos descendientes de las prostitutas. Los niños de raza mixta fueron excluidos y repudiados de la sociedad coreana, cuyo orgullo nacional se basaba en mantener una línea racial pura. Los bebés resultantes del mestizaje eran objeto de discriminación social desde su nacimiento (Lim 2017, 243). Ser hijo de una prostituta conllevaba el estigma de una inmediata identificación con sus orígenes de inmoralidad; su desprestigio trascendía incluso a los que los cuidaban, de ahí que muchos acabaran en orfanatos o abandonados.

En Chinatown, Maggie tiene una hija de cinco años, Jenny, fruto de una antigua relación con un soldado blanco. Tras el asesinato, la tía de la niña acude a recoger las pertenencias de la difunta, pero se niega a responsabilizarse de la niña, que acaba abandonada en un orfanato. Esta parte de la historia vuelve a mostrar el rechazo de las otras mujeres a ser relacionadas con una wianbu, aunque ello supusiera el abandono de una vida inocente. Y de nuevo surge otro ejemplo de otredad: los mestizos huérfanos no son aceptados por el resto de la sociedad. En el siguiente fragmento extraído de Chinatown, la abuela rechaza a la niña: 
-Es una bestia salvaje - dijo la abuela con la misma mirada llena de odio con la que miraba a los animales con pelo, lo que ella más odiaba. También observaba a Jenny sentada a veces afuera de la puerta o en la terraza, como si fuera una cosa ajena (Oh 2002, 231).

Este relato ayuda a imaginar la dura vida de estos huérfanos desamparados: sin hogar, indefensos, hambrientos, discriminados por ser fruto de la obscenidad y por haber heredado rasgos de sus padres occidentales. Los mestizos acabaron pagando por la deshonrosa labor de sus madres. Eran sujetos completamente desplazados por el pueblo y su existencia fue el retrato vivo de la otredad más cruel ejercida por la sociedad de la época.

\section{Conclusión}

En los convulsos tiempos de la posguerra, las mujeres se adaptaron a los cambios y buscaron con anhelo un mejor futuro, a pesar de vivir en una sociedad patriarcal y autoritaria. Su lucha y su perseverancia, sin embargo, fueron insuficientes frente a la cruda realidad. Mención especial merecen las injusticias y los constantes abusos que sufrieron las wianbu. Estas mujeres se vieron empujadas a ejercer la prostitución como fuente de recursos para su supervivencia y la de sus familias. Tuvieron que aguantar el estigma del desprecio de la población local. Se enfrentaron al desamparo y la marginación por la falta de protección legal ante la violencia que los “otros” ejercían sobre ellas. Finalmente, sus descendientes heredaron la mancha del adulterio de sus madres.

En los dos textos seleccionados se muestran visiones opuestas: Chinatown intenta revelar la realidad desde dentro, mostrando las miserias y la discriminación que sufrían las prostitutas, mientras que el informe policial, con una cuidada selección lingüística, pretende maquillar la prostitución y los conflictos que genera. La novela destapa una realidad que estaba oculta; el informe la esconde. 
Aproximadamente cuarenta años después, presenciamos el movimiento social Me Too de reivindicación feminista. El desencadenante principal en Corea del Sur ha sido el sinfín de incidentes no resueltos de violencia sexual sufridos por mujeres con posiciones laborales o sociales de inferioridad respecto a la de sus agresores (Kim 2019, 3). Del mismo modo, H.-S. Kwon (2018, 259-260) critica la cosificación del cuerpo de las mujeres y que los agresores aprovechen su poder y la supremacía de su estatus social. Es decir, la otredad de las víctimas es un tema relevante y todavía representa un reto para nuestra sociedad. El actual movimiento nos hace revisar el concepto de wianbu, invisible pero aún presente.

\section{Referencias}

Al-SAIDI, Afaf Ahmed Hasan. 2014. "Post-colonialism Literature the Concept of Self and the Other in Coetzee's Waiting for the Barbarians: An Analytical Approach". Journal of Language Teaching and Research 5, núm. 1: 95-105. https://doi.org/10.4304/ jltr.5.1.95-105

Chaure, Desirée Nair. 2018. "El rol de la mujer en Corea: cambios y continuidades”. En e-Corea:procesos políticos, económicos y sociales en la península coreana, compilado por Luciano Damián Bolinaga y Bárbara Bavoleo, 161-181. Buenos Aires: Teseo.

CHOI, Yisook. 2015. "Commercialization of Korean Press Industry and the Gender Politics of Women's Pages in the 1960-1970's”. Korean Journal of Journalism and Communication Studies 59, núm. 2: 287-323. https://www.dbpia.co.kr/journal/articleDet ail?nodeId $=$ NODE06291863\#

FaIRClOUgh, Norman. 1992. Discourse and Social Change. Cambridge: Polity Press.

FAIRCLOUGH, Norman. 2012. "Critical Discourse Analysis". International Advances in Engineering and Technology 7: 452-487. http:// scholarism.net/FullText/2012071.pdf

GuAhK, SangSoon. 2018. “A Study on Oh Jeong-hee's Novels”. The Society for Korean Language E Literary Research 46, núm. 2, 
319-337. https://www.dbpia.co.kr/Journal/articleDetail?node $\mathrm{Id}=$ NODE08956014\#

Hwang, Namyeob. 2015. "The Racial Discrimination in Chinatown:

Disappearing Moon Cafe and Chinatown". The Journal of East-

West Comparative Literature 34: 191-218. https://www.dbpia. co.kr/journal/articleDetail? nodeId = NODE06575386\#

Hynes, H. Patricia. 2004. "On the Battlefield of Women's Bodies: An Overview of the Harm of War to Women". Women's Studies International Forum 27, núm. 5-6: 431-445. https://doi. org/10.1016/j.wsif.2004.09.001

JANG, So-jin. 2006. "A Boy and a Girl, the Growth of Deviation and Fixation". EOMUNYEONGU 34, núm. 4: 303-329. https:// academic.naver.com/article.naver?doc id $=12975410$

JeONG, Jai Rim. 2006. "Research about Image Memory of Oh Jeong hui's Novel”. Comparative Korean Studies 14, núm. 1: 203226. https://www.kci.go.kr/kciportal/ci/sereArticleSearch/ ciSereArtiView.kci?sereArticleSearchBean.artiId = ART001015340

Joo, Jiyoung. 2016. "The World Represented as 'Chinatown', and the Identity of Women -Oh Jeonghee's 'Chinatown'”. Journal of Literary Creative Writing 15, núm. 2: 77-115. https://www. kci.go.kr/kciportal/ci/sereArticleSearch/ciSereArtiView. kci?sereArticleSearchBean.artiId = ART002143330

KIM, Bo-Hwa. 2019. "A Study of Meaning of the MeToo Movement in 2018, Korea through Analyzing Sexual Violence Counseling Journals". Issues in Feminism 19, núm. 2: 3-43. https://doi. org/10.21287/iif.2019.10.19.2.3

$\mathrm{KIM}, \mathrm{Ho}$ Sik 김호식. 1970. 경기 감축 기지촌 [Reducción de negocios en Gijichon]. JoonAng Ilbo 중앙일보. https://news.joins.com/ article/1246065

KIM, Jihye. 2017. "Embodying and Performing Female Identities in Oh Jung-hee's Novels: Focusing on The Garden of Childhood, Chinatown, and An Evening Game". Issues in Feminism 17, núm. 2: 93-126. https://doi.org/10.21287/iif.2017.10.17.2.93

KIM, Won. 2015. "Critical Juncture of Isolating U.S. Camptown: Special Districts, Korean American Friendship Society and U.S. Camptown Purification Movement”. Critical Review of History 112: 153-185. https://www.dbpia.co.kr/Journal/articleDetail?n odeId = NODE06505976\# 
Kwon, Hyang-Sook. 2018. "A Philosophical Consideration of Me Too Movement in Korea”. The Journal of Humanities 39, núm. 4: 259-285. https://www.dbpia.co.kr/journal/articleDetail?nodeI $\mathrm{d}=$ NODE07567895\#

LEE, Hyun Shik. 2008. "A Study on Oh, Cheong-hee's Chinese Street". Dongnam Journal of Korean Language and Literature 1, núm. 26: 73-91. https://scienceon.kisti.re.kr/srch/selectPORSrchArticle. do? $\mathrm{cn}=\mathrm{ART}$ T0 1575313

LeE, Mihwa. 2018. "A Study on the Types of Women's Images in Oh, Jeong-hee's The Chinese Street”. Foreign Literature Studies 69: 143-164. https://doi.org/10.22344/fls.2018.69.143

LeE, $\mathrm{Na}$ Young. 2006. “The Construction of U.S. Camptown Prostitution in South Korea: Trans/Formation and Resistance". Tesis doctoral. University of Maryland. http://hdl.handle. net/1903/4162

LeE, Na Young. 2008. "Postcolonial Reading on Military Prostitutes in South Korea: Gendered Nationalism and Politics of Representation”. Journal of Korean Women's Studies 24, núm. 3: 77-109. https://www.dbpia.co.kr/Journal/articleDetail?nodeId=NO DE01066823\#

LeE, Na Young. 2013. “Global/Local Gender Order and Women's Body in Korea: Japanese Military 'Comfort Women' and U.S. Camptown Prostitutes ('Yanggongju')”. Dong Bang Hak Chi 161: 3-38. https://www.dbpia.co.kr/Journal/articleDetail?nod eId $=$ NODE08840596\#

LEE, Sookin. 2007. "Criticism of the Colonization of Korean Women (Feministic) Knowledge - Strategy of De-Westernization and De-Confucianism”. Oriental Philosophy 28: 197-227. https://doi. org/10.19065/japk..28.200712.197

León Manríquez, José Luis y Juan Felipe López Aymes. 2009. “Corea del Sur”. En Historia minima de Corea, coordinado por José Luis León Manríquez, 147-190. México: El Colegio de México.

LIE, John. 1995. "The Transformation of Sexual Work in 20thCentury Korea”. Gender and Society 9, núm. 3: 310-327. https:// doi.org/10.1177/089124395009003004

LIM, Eun Hee 임은희. 2017. 현 대소설에 나타난 ‘양공주' 모티프 의 다문화적 사유와 타자성 [A Study on the Multicultural Thinking and Otherness of 'Yanggongjus' Motif in Modern Novels]. 한국문예비평연구 Study of Korean Modern Lite- 
rary Criticism, núm. 54: 241-267. https://doi.org/10.35832/ kmlc..54.201706.241

Moon, Katharine H. S. 1997. Sex among Allies: Military Prostitution in U.S.-Korea Relations. Nueva York: Columbia University Press.

$\mathrm{OH}$, Jung Hee 오정희. 2002. 중국인 거리 [Chinatown]. En 스무 살을 위한 페미니즘 소설 [Novelas feministas para personas de 20 años], editado por Jeong Hui Lee 이정 희, 214-245. Seúl: Ch'ŏngdong Kŏul 청동거울. https://jdlib.sen.go.kr/jdlib/ intro/search/detail.do?vCtrl $=200811286 \&$ isbn $=8988286669$ \&menu $\mathrm{idx}=4$

Он, Yang-Ho. 2012 . "A Study on Incheonhak as a Literary Geography”. Journal of Incheon Studies 1, núm. 17: 225-254. https://www.dbpia.co.kr/journal/articleDetail?nodeId = NO DE06590623\#

$\mathrm{OH}$, Youn-Ho. 2007. "A Study on Diaspora Imagination of The Chinese Street”. The Society for Korean Language E Literary Research 35, núm. 4: 265-288. https://doi.org/10.15822/skllr.2007.35.4.265

PARK, Jeong-Mi. 2013. "Paradoxes of Gendering Strategy in Prostitution Policies: South Korea's 'Toleration-Regulation Regime', 1961-1979”. Women's Studies International Forum 37: 73-84. https://doi.org/10.1016/j.wsif.2012.10.008

PARK, Jeong-Mi. 2015. “A Historical Sociology of the Korean Government's Policies on Military Prostitution in U.S. Camptowns, 1953-1995: Biopolitics, State of Exception, and the Paradox of Sovereignty under the Cold War". Korean Journal of Sociology 49, núm. 2: 1-33. https://doi.org/10.21562/ kjs.2015.04.49.2.1

PARK, Jong Hyun. 2016. "Silent Diaspora: The Representations of Women in Military Camp Town and Children of Mixed Blood". Journal of Basic Design E Art 17, núm. 1: 231-243. https://www. kci.go.kr/kciportal/ci/sereArticleSearch/ciSereArtiView. kci?sereArticleSearchBean.artiId = ART002088150

Policía del distrito de Yongsan 용산경찰서장 공문. 1971. 미국접 객업에 종사하는 여러분에게 [A ustedes, que se encuentran prestando el servicio en pro del compromiso con el negocio de la hospitalidad para el ejército de Estados Unidos]. https://news. sbs.co.kr/news/endPage.do?news_id=N1004676382

Ríos, Enrique. 2001. "Aproximación a los medios de comunicación en Corea del Sur”. En Corea Frente a los Desafíos del Siglo XXI: 
Primer Simposio Internacional sobre Corea. Madrid, 29 y 30 de noviembre de 2000, coordinado por Alfonso Ojeda, Ernesto de Laurentis y Álvaro Hidalgo, 71-82. Madrid: Centro Español de Investigaciones Coreanas.

SoH, C. Sarah. 2008. The Comfort Women: Sexual Violence and PostColonial Memory in Korea and Japan. Chicago: The University of Chicago Press.

Van Dijk, Teun A. 1998. Ideology: A Multidisciplinary Approach. Londres: Sage.

VAN DijK, Teun A. 2001. "Multidisciplinary CDA: A Plea for Diversity”. En Methods of Critical Discourse Analysis, editado por Ruth Wodak y Michael Meyer, 95-120. Londres: Sage.

Morgan Mok-Won Park: es investigadora y profesora lectora del Área de Estudios de Asia Oriental del Departamento de Filología Moderna de la Universidad de Salamanca. Es doctora en lingüística teórica y aplicada por la Universidad Complutense de Madrid. Su carrera profesional ha consistido en investigación, docencia, traducción e interpretación.

$$
\begin{array}{r}
\text { https://orcid.org/0000-0002-5064-474X } \\
\text { morganpark53@gmail.com }
\end{array}
$$


